\title{
Development and validation of a short runtime method for separation of trace amounts of 4-aminophenol, phenol, 3-nitrosalicylic acid and mesalamine by using HPLC system
}

\author{
Seyyed Amir Siadatia ${ }^{*}$, Mohammad Amin Rezvanfar ${ }^{a, b, c}$, Meghdad Payab and Abolghasem \\ Beheshti ${ }^{a, d}$
}

${ }^{a} R \& D$ Department, Tofigh Daru Research and Engineering Company, TPICO, Tehran, Iran

${ }^{b}$ Department of Toxicology and Pharmacology, Faculty of Pharmacy, and Pharmaceutical Sciences Research Center, Tehran University of Medical Sciences (TUMS), Tehran, Iran

${ }^{c}$ Department of Toxicology and Pharmacology, School of Pharmacy, Tehran University of Medical Sciences, Tehran, Iran

${ }^{d}$ Department of Chemistry, Payame Noor University, Tehran, Iran

\section{H R O N I C L E}

Article history:

Received August 1, 2020

Received in revised form

November 29, 2020

Accepted December 14, 2020

Available online

December 14, 2020

Keywords:

Isocratic separation

HPLC

Mesalamine

Trace amounts

Salicylic acids

\begin{abstract}
A B S T R A C T
In a short runtime process (about 6 minutes), with isocratic method without need of reconditioning before the next run, a mixture of some molecules containing 4-aminophenol (4APh), phenol, 3-nitrosalicylic acid (3NSA), and mesalamine (5-aminosalicylic acid) (MLZ), were separated and detected by means of HPLC/UV-Vis system. The resolutions of the separated and sharp peaks referring to the mentioned compounds were at least more than 2 , and the tailing factor (TF) was about 1 , even in $1 \mu \mathrm{g} / 1$ ( $\mathrm{ppm}$ ) concentration. In order to optimize the method, the effects of the $\mathrm{pH}$, eluent percentage, flow rate, and buffer concentration, and wavelength on the chromatogram were investigated. Also, the results of the validation processes showed that the method trustable to be used in laboratories.
\end{abstract}

(C) 2021 Growing Science Ltd. All rights reserved.

\section{Introduction}

In one hand, separation and detection of trace amounts of phenolic or benzylic acid compounds are important both in medicinal (human metabolism) ${ }^{1}$ and environmental chemistry, ${ }^{2}$ and in the other hand, determination of one part per million (PPM or $\mu \mathrm{g} / \mathrm{l}$ ) concentration of some of the aminophenols in Mesalazine is crucial in pharmacology. ${ }^{3}$ Due to these requirements, designing suitable methods for Related Impurity Analysis (RIA), ${ }^{4,5}$ Assay Analysis (AA), ${ }^{6}$ Residual Solvent Analysis (by gas chromatography) (RSA), ${ }^{7}$ and other instrumental analysis for MLZ and related compounds were designed, and widely applied. Song and colleagues designed an electrochemical method for RIA of MLZ using a C8 column, with the mobile phase of PDP, and sodium 1-octanesulfonic acid sodium salt (OSA) buffer solution at $\mathrm{pH}$ 2.2, methanol, acetonitrile (890:80:30) and methanol via gradient elution. A glassy carbon electrode and a silver-silver chloride electrode were used for the electrochemical detection, respectively. ${ }^{4}$ The pharmacopeias like UPS have proposed some related HPLC methods for RIA of MLZ using UV detectors. ${ }^{7}$ Sometime during synthesis of organic compounds ${ }^{8-10}$ a number of 
organic impurities like precursors, intermediates, or degrading products are formed that is, the RIA is very important for detection of probable impurities. An example for HPLC assay method was a chromatography condition containing a SuperPac Sephasil C18 column $(5 \mu \mathrm{m} ; 250 \mathrm{~mm}, 4.0 \mathrm{~mm}$; SuperPac Sephasil). The samples were isocratically eluted with a $30 \mathrm{mM}$ PDP buffer and methanol with a volume ratio of $70: 30$. The buffer was also contained $25 \%$ tetrabutylammonium hydrogen sulphate, with the flow rate of $1.0 \mathrm{~mL} / \mathrm{min}$. The UV detection at $254 \mathrm{~nm}$ was performed. ${ }^{6}$

In the case of choosing methods for use, when applying the gradient HPLC methods, after the end of the running process, the operator must consider a time for re-conditioning of the system at the zero point of elution (unlike isocratic methods). ${ }^{11}$ Therefore, sometimes operators (especially in industries) and commercially users prefer the isocratic method that does not need for re-conditioning. But, use of isocratic methods may results in poor separation, low resolution of the peaks, and long running time. ${ }^{12}$ Thus, in many cases, method designers have to develop gradient methods instead of isocratic ones. In an equal condition, when the runtime, resolutions, and other parameters both for gradient and isocratic methods are the same, the isocratic method maybe preferred especially in industries or in laboratories. ${ }^{13}$ On the other hand, methods with the ability of separating mixed standards are more efficient and more preferred than methods which could separate only one or two standards. If a method could separate a mixed of a number of standards by a single run instead of preceding several runs for a number of single standards, there will be a great saving in time, energy, and solvent. Thus, designing methods which separate and detect a mixed of standards by a single run, with sharp, symmetric and high resolution peaks, would be useful and interested both for laboratories and industries. ${ }^{14}$

Due to above mentioned debates, in this research, we have designed a short (in 6 minutes), single run isocratic method that is able to separate 4-aminophenol (4APh), phenol, 3-nitrosalicylic acid (3NSA), and mesalamine (5-aminosalicylic acid) (MLZ) resulting in high resolution and sharp peaks. In the optimized chromatography condition, a C18, end-capped $(250 \times 4.6) \mathrm{mm}, 5 \mu \mathrm{m}$ liquid chromatography column was used for the analysis. The mobile phase was a mixture of (buffer:methanol) with a ratio of (60:40). The buffer was prepared by dissolving $1.1 \mathrm{~g}$ of $(5 \mathrm{mM}) \mathrm{OSA}$ in 1 liter of water to $\mathrm{pH}=2.70$ with OPA. The isocratic elusion with a flow rate of $1.5 \mathrm{ml} / \mathrm{min}$ was set for the method. In addition, a UV detector on the wavelength of $210 \mathrm{~nm}$ was used for recording the chromatograms. The column oven temperature was maintained at $40^{\circ} \mathrm{C}$ and the volume of injection was $20 \mu \mathrm{L}$. Also, the mobile phase was used as the diluent. The sample solutions were prepared as following.

\section{Results and Discussion}

In the present project, at the first step, several parameters were optimized to reach the best conditions and then, the limit of detection (LD), linearity, and some other validation parameters were investigated. Following sections describe the process of optimization and further investigations.

\subsection{Effect of various parameters}

In order to find the best chromatography condition with higher possible separation, resolution, and linearity, and also to reach to the lowest limit of detection, several parameters containing the concentration of PDP, and OSA salts, $\mathrm{pH}$ of the buffer, organic solvent percentage, flow rate, and wavelength were optimized. The results showed that by optimizing each of the mentioned parameters, higher resolution, and better separation could be reached.

\subsubsection{Effect of PDP}

At the beginning, we prepared a buffer by dissolving 10mM PDP (both as pH controller), and $10 \mathrm{mM}$ OSA as column modifier (for better interactions between the stationary phase and the components of the mixed standard) to different $\mathrm{pHs}$ optimized by OPA as the acid agent. The mobile 
phase contained of $60 \%$ of buffer and $40 \%$ of methanol as organic solvent. Then, in the first step, we changed the concentration of PDP from $20 \mathrm{mM}$, to $10 \mathrm{mM}, 5 \mathrm{mM}, 1 \mathrm{mM}$, and finally elimination of the salt from above mentioned buffer. The results showed that decreasing the PDP salt in buffer or even elimination of that (it means just OSA is used), could result in better separation and higher resolution of all four peaks. While using that salt increase the tailing factor of the peaks. On the other hand, elimination of PDP and use of only the OSA salt, led the method to be easier and cheaper. Due, to these, the PDP was eliminated from buffer and all of the optimization processes were performed by OSA.

\subsubsection{Effect of $p H$}

The results showed that changes on the $\mathrm{pH}$ strongly effects on the chromatogram resolution, peak separation, and the RT of those. In order to optimize the $\mathrm{pH}$ value, we fixed all mentioned parameters and only changed the $\mathrm{Ph}$. At the first step, the $\mathrm{pH}$ was set on about 5.5. At this $\mathrm{pH}$, peaks 1 , and 2 appeared about 1.7 min and 2.2 min respectively, while peaks 3, and 4 appeared in one peak. By decreasing the $\mathrm{pH}$ to 3.3 , the RT of peaks 1 , and 2 changed to about $1.8 \mathrm{~min}$ and $2.4 \mathrm{~min}$, while the unique peak which represented peaks 3 , and 4 broke in two overlaid peaks (Figure 1).

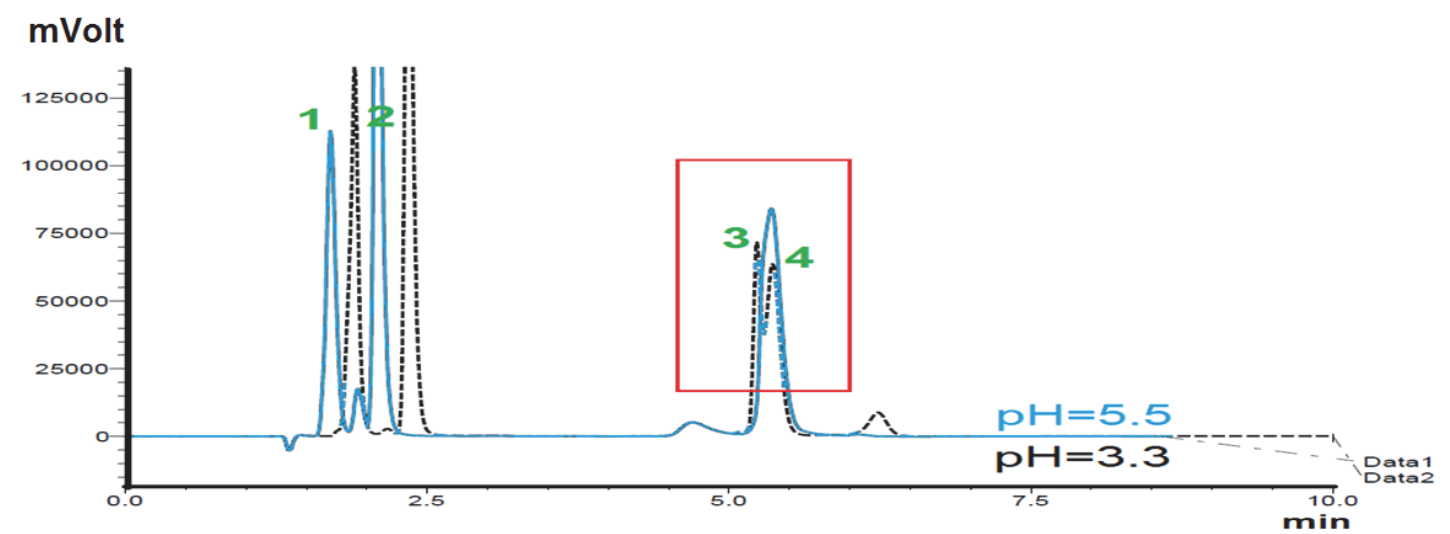

Fig. 1. The effect of $\mathrm{pH}=5.5$, and $\mathrm{pH}=3.3$ on the $\mathrm{RT}$ of peaks, and splitting between peaks 3 , and 4 .

In addition, we changed the $\mathrm{pH}$ to different values. Fig. 2 shows our results reached by $\mathrm{pHs}$ about $3.3,2.7$, and 2.5. The results showed that by decreasing the $\mathrm{pH}$ values from 3.3 to 2.5 , the splitting between peaks 3 , and 4 increase and also peaks 1 , and 2 moves to upper RTs. Due to these, $\mathrm{pH}=2.7$ was selected as the optimum $\mathrm{pH}$, and in further experiments, other parameters were being optimized.

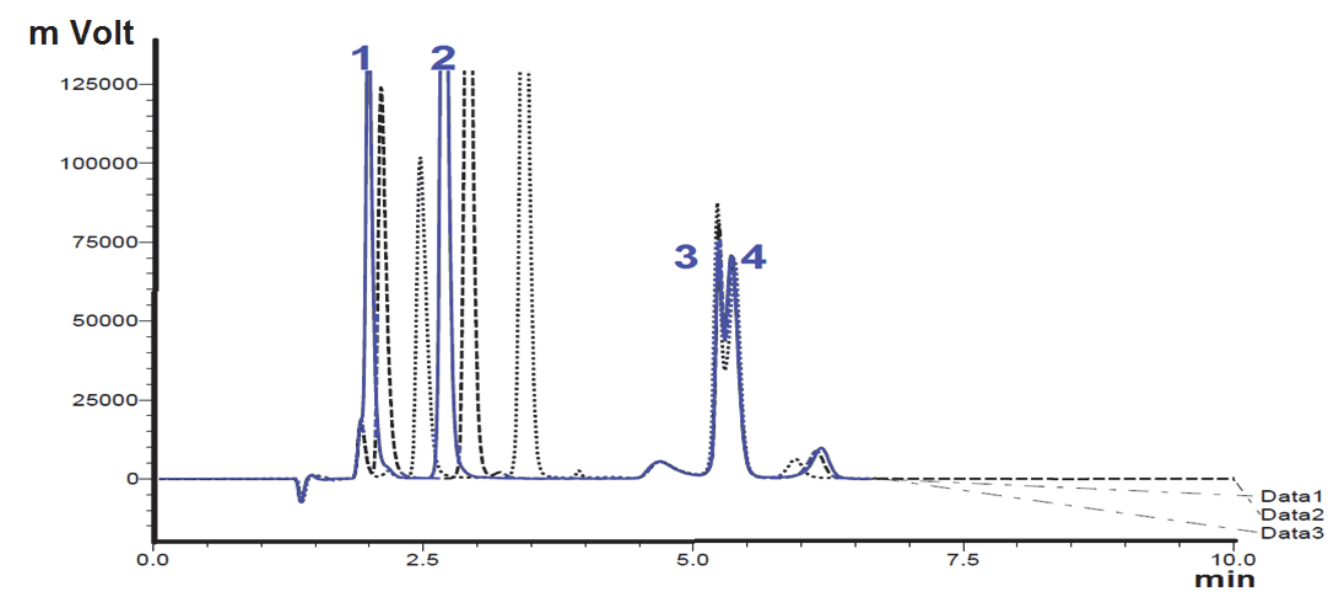

Fig. 2. Effect of $\mathrm{pH}$ on the RT of peaks, and splitting of peaks 3, and 4. Blue line, dashes, and dots, represent $\mathrm{pH}=3.3, \mathrm{pH}=2.7$, and $\mathrm{pH}=2.5$. 


\subsubsection{Effect of methanol percentage}

All of the liquid chromatography parameters were being fixed, and the percentage of methanol was the only parameter which was changed. As shown in Fig. 3, by changing the methanol percentage from $40 \%$ (blue line) to $45 \%$ (dash) and 50\% (dots), the separation of the peaks 3 , and 4 , were significantly better; while, in the case of peaks 1 , and 2 , it resulted in overlaying of those two. Moreover, by increasing the methanol percentage to $50 \%$, peak 1 was linked to the injection peak (red circle).

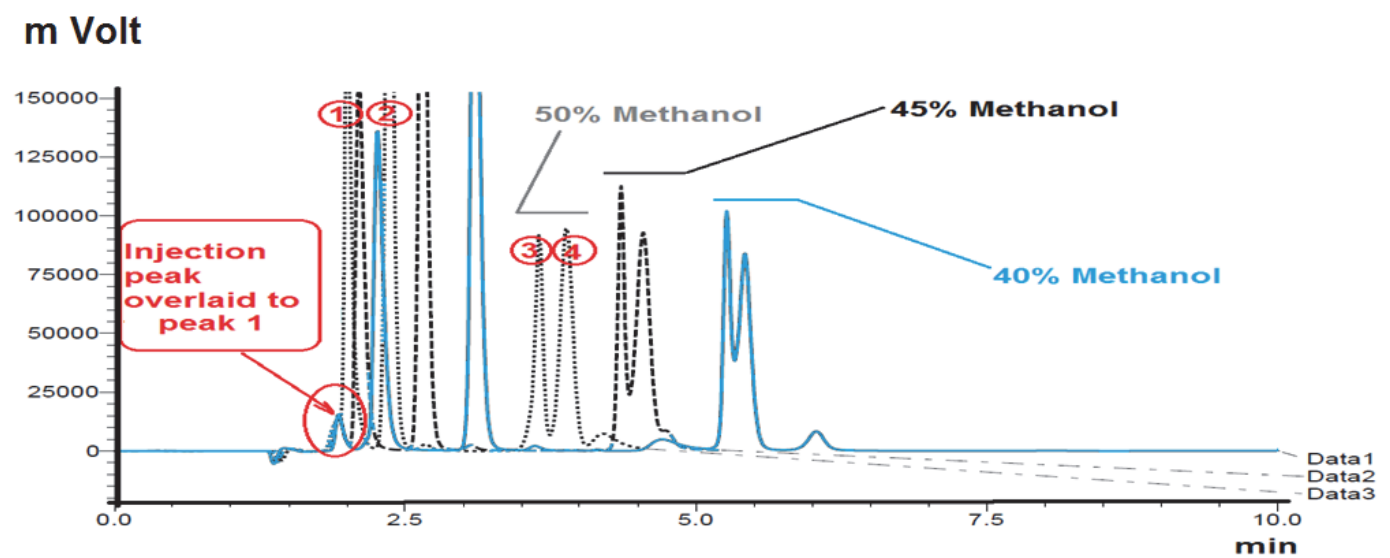

Fig. 3. Change of methanol concentration results in change of separation between peaks 3 , and 4 .

Also, another experiment showed that using $37 \%$ of methanol led to a very weak separation of peak 3 , and 4 compared to $40 \%$. In the following section, the experiments showed that by selecting the methanol percentage on $40 \%$, and changing OSA concentration in buffer, the separation of peaks 3 , and 4 became better, while it does not lead to a bad effect on peaks 1, and 2 separations (Fig. 4).

\subsubsection{Effect of OSA concentration}

As shown in Fig. 4, the concentration of OSA in buffer, directly effects on the peak separations and also on the position of those. Somehow, by using the $10 \mathrm{mM}$ of OSA in buffer (dots), peaks 1 , and 2 are pretty separated, while peaks 3 , and for (RT about $5.5 \mathrm{~min}$ ) are overlaid. Also, by using $2.5 \mathrm{mM}$ of OSA (blue line), peaks 3, and 4 are separated, but peaks 1 , and 2 are overlaid. Then, we used $5 \mathrm{mM}$ of OSA in buffer (green dashes) and observed that both groups of peaks (peaks 1 and 2, and peaks 3, and 4) are separated. Thus, we choose $5 \mathrm{mM}$ of OSA in buffer concentration as the optimum for this parameter.

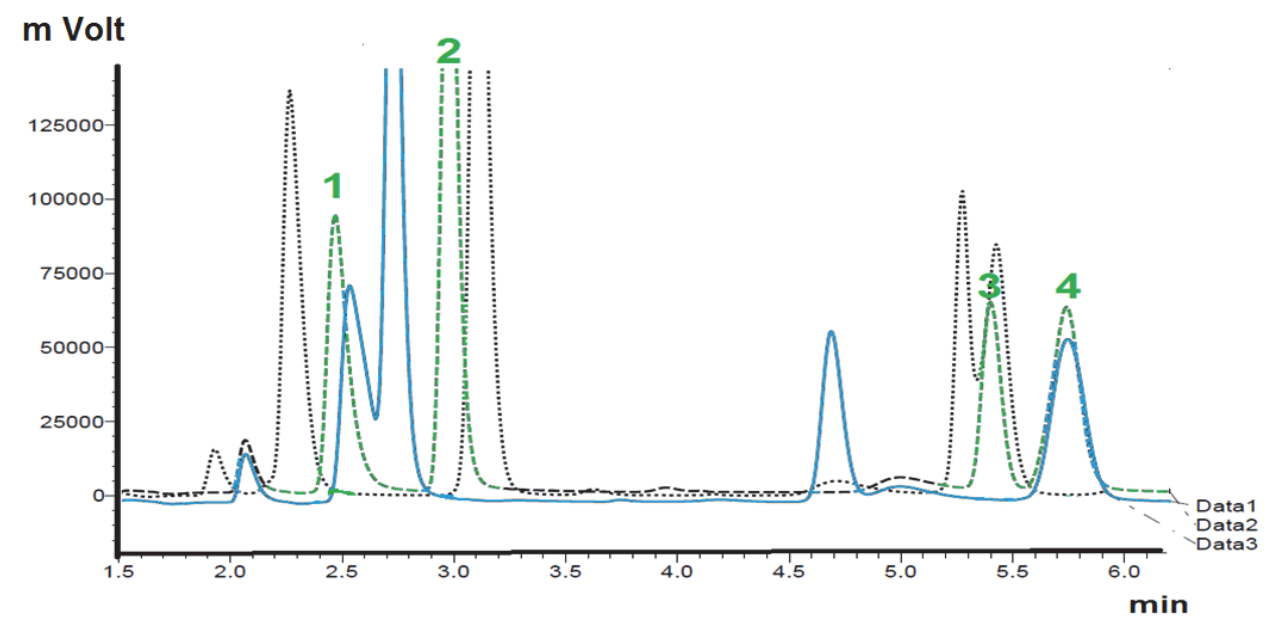

Fig. 4. The effect of OSA concentration in buffer solution, on the peak separation 


\subsubsection{Effect of flow rate change}

As shown in Fig. 5, the flow rate changes from $1.5 \mathrm{ml} / \mathrm{min}$ to $1.3 \mathrm{ml} / \mathrm{min}$ showed that it just changes the RT of the peaks and it does not lead to a better separation of peaks 3 , and 4 (4APh, and Phenol, respectively).

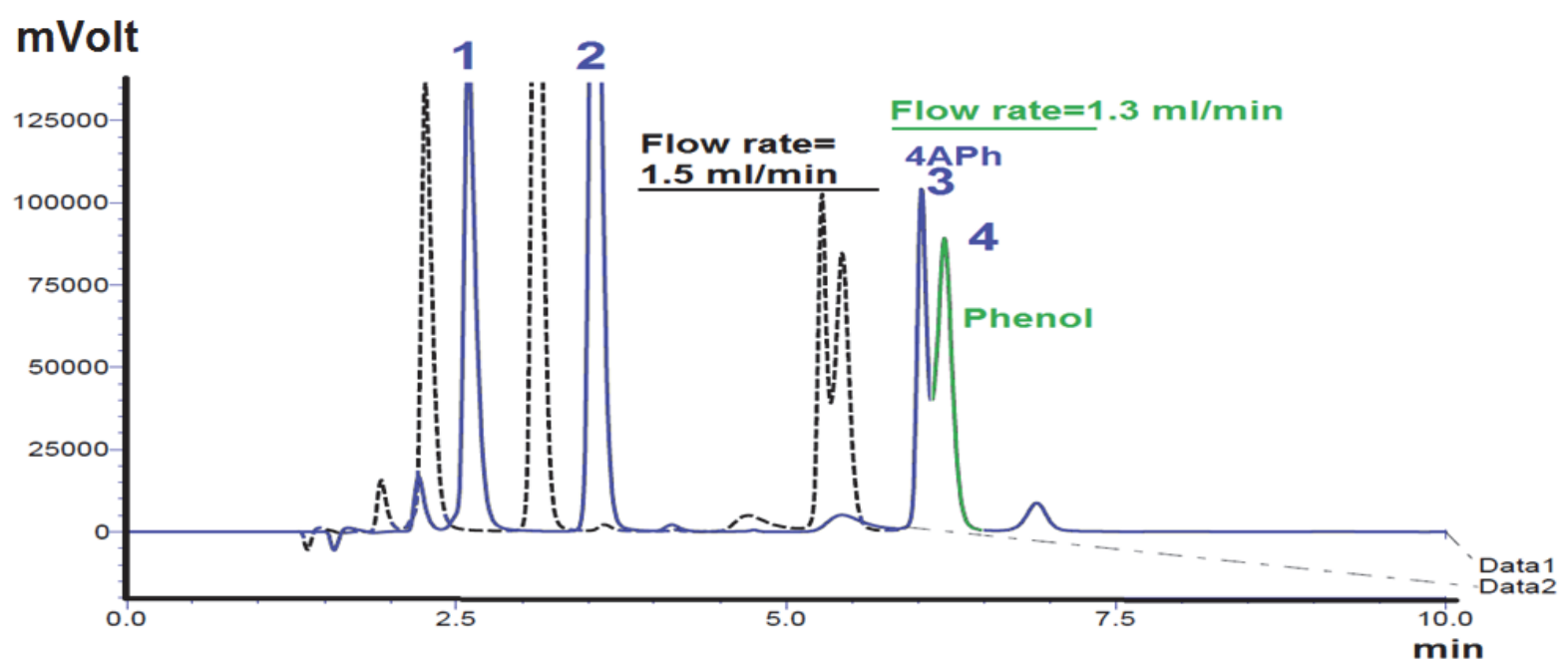

Fig. 5. The effect of flow rate change from $1.5 \mathrm{ml} / \mathrm{min}$ to $1.3 \mathrm{ml} / \mathrm{min}$

\subsubsection{Effect of the wavelength}

As shown in Fig. 6, by decreasing the number of the wavelength from $240 \mathrm{~nm}$ to $210 \mathrm{~nm}$, the intensity of the peaks increases. Somehow, at $210 \mathrm{~nm}$, all four peaks could be detected strongly. At 220 $\mathrm{nm}$, the area of peaks 1 , and $2(\mathrm{RT}=2.5$, and 3 ) significantly decrease, while it is not observed for peaks 3 , and $4(\mathrm{RT}=5.4$, and $5.7 \mathrm{~min})$. in addition, at $230 \mathrm{~nm}$, and $240 \mathrm{~nm}$, a considerable decrease is observed for all peaks. It is notable that at $240 \mathrm{~nm}$, peaks 3 and 4, could not be detected in practice. Since at wavelengths lower than $210 \mathrm{~nm}$, the UV cutoff of elution may affect on the efficiency of the optimized method, the $210 \mathrm{~nm}$ was selected as the optimum wavelength.

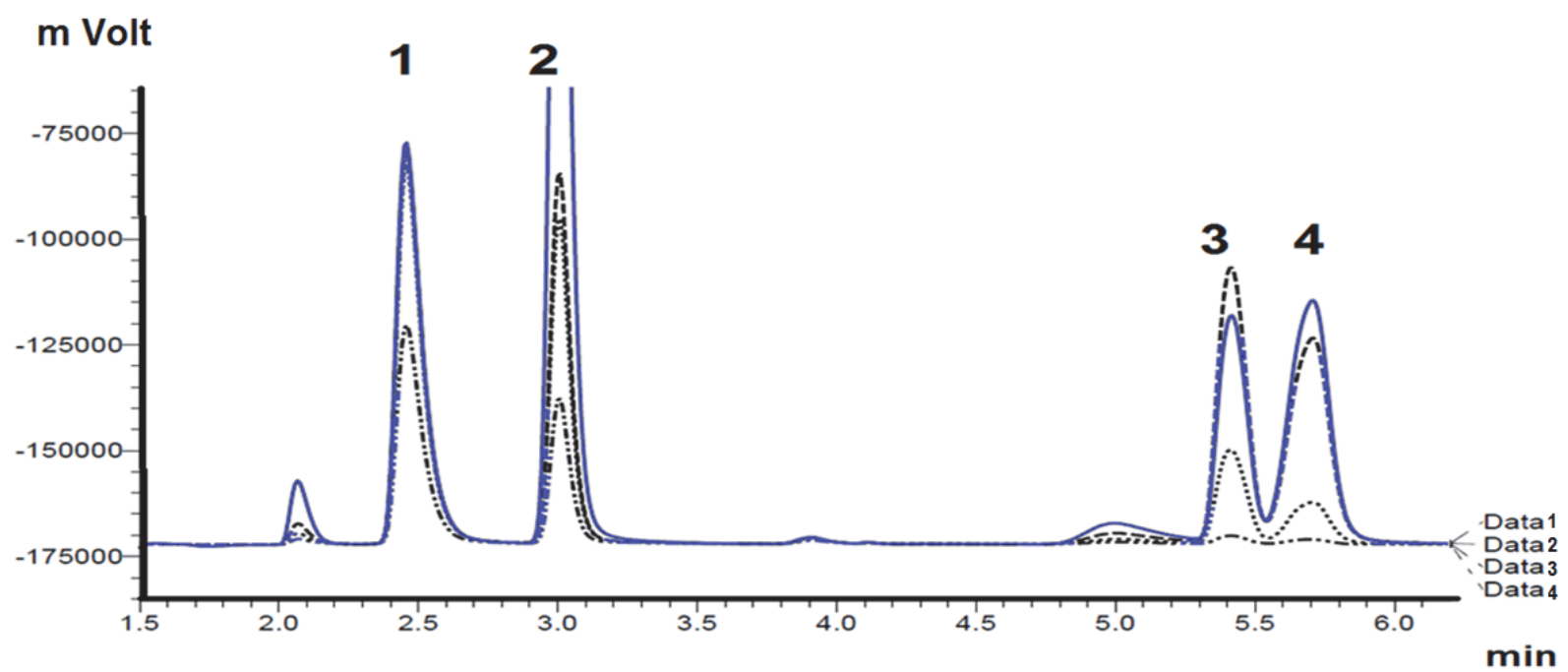

Fig. 6. The effect of the recording wavelength, on the peak intensity (mV.min). The blue gram (Data 1) is $210 \mathrm{~nm}$, and the Data 2, Data 3, and Data 4, are 220nm, $230 \mathrm{~nm}$, and $240 \mathrm{~nm}$, respectively. 


\subsection{Validation of the optimized method}

In the optimized chromatography condition, a C18, end-capped $(250 \times 4.6) \mathrm{mm}, 5 \mu \mathrm{m}$ liquid chromatography column was applied for the solid-liquid phase chromatography separation. The buffer was prepared by dissolving $5 \mathrm{mM}$ octane-1-sulfunic acid sodium salt in water to $\mathrm{pH}=2.70$ by OPA (then filtered by a $0.45 \mu \mathrm{m}$ filter). The mobile phase was a mixture of (buffer/methanol) with a ratio of $(60 / 40)$. The isocratic elution was operated at a flow rate of $1.5 \mathrm{ml} / \mathrm{min}$. The oven temperature was about $25^{\circ} \mathrm{C}$, and the chromatograms were recorded on $210 \mathrm{~nm}$. By setting these parameters, all four peaks referring to 3-nitrosalicylic acid, mesalamine, 4-aminophenol, and phenol (respectively), were separated and detected with a low tailing factor and a good resolution.

\subsubsection{Linearity study}

It is obvious that the linearity of an analytical method is its ability (within a considered range) to give results which are directly (or by a mathematical transformation), proportional to the concentration of the sample (or standard). The linearity of the present procedure was assessed by injecting the solutions of a mixed standard containing four related compounds, listed in Table 1 (each solution was injected three times). A linear calibration curve was prepared by plotting the average analytical signal versus mixed standard concentration (Fig. 7). A regression line was calculated by the procedure of least squares.

Table 1. The areas (mV.min) of four peaks referred to 3NSA, MLZ, 4APh, and Phenol, respectively, obtained by recording the mixed standard solutions from $0.1 \mathrm{ppm}$ to $10 \mathrm{ppm}$

\begin{tabular}{ccccc}
\hline Concentration & 3NSA & MLZ & 4APh & Phenol \\
\hline $\mathbf{0 . 0 1} \mathbf{~ p p m}$ & 220 & 1237 & - & - \\
$\mathbf{0 . 1} \mathbf{~ p p m}$ & 4278 & 11779 & 2269 & 9977 \\
$\mathbf{0 . 5} \mathbf{~ p p m}$ & 24657 & 63664 & 16253 & 30417 \\
$\mathbf{1} \mathbf{~ p p m}$ & 58478 & 123849 & 34800 & 57494 \\
$\mathbf{5} \mathbf{~ p p m}$ & 299201 & 589663 & 181401 & 274698 \\
$\mathbf{1 0} \mathbf{~ p p m}$ & 626286 & 1190157 & 380162 & 564115 \\
$\mathbf{R}^{\mathbf{2}}$ & 0.999 & 0.999 & 0.999 & 0.999 \\
\hline
\end{tabular}

The linearity curve given in Fig. 7, indicates that the coefficient of determination $\left(\mathrm{R}^{2}\right)$ for each of four standards was 0.999 which showed a good linearity in the concentration range of $0.1 \mathrm{ppm}$ to 10 $\mathrm{ppm}$. Also, in the case of MLZ, the concentration ranges begin from $0.01 \mathrm{ppm}$ to $10 \mathrm{ppm}$.

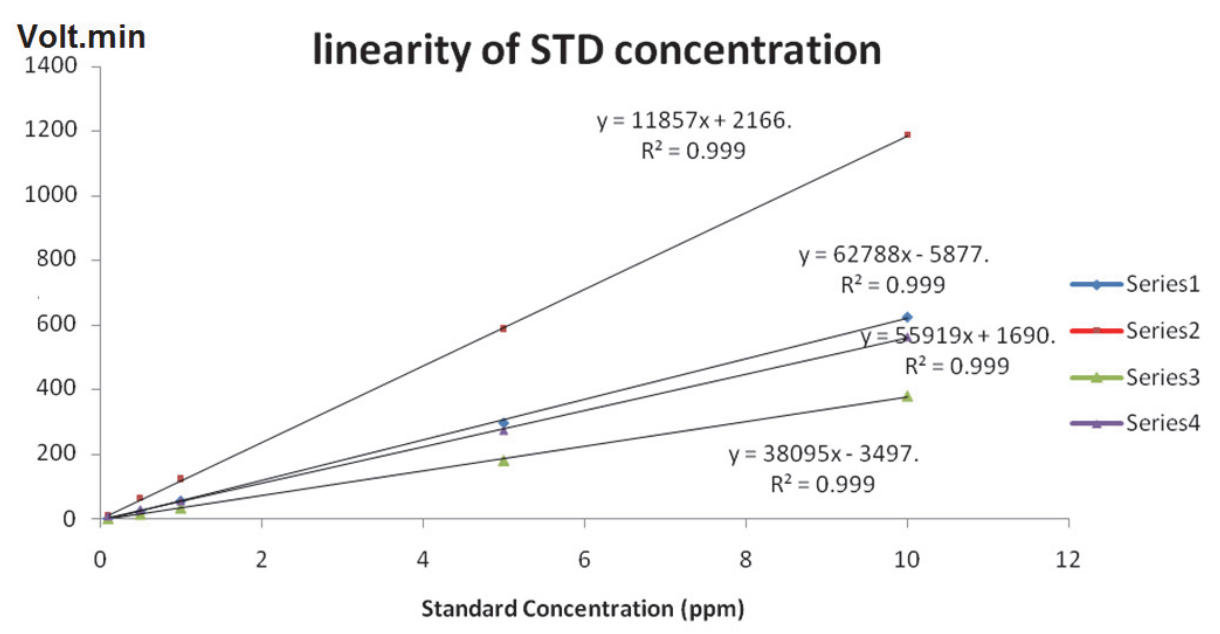

Fig. 7. The calibration curve for the linearity study referred to four considered standards. The series 1 to 4, represent 3NSA, MLZ, 4APh, and Phenol, respectively. 


\subsubsection{Limit of Detection (LD) and Quantification limit $(Q L)$}

As given in literature, the limit of detection (LD) of an individual analytical method is the lowest possible amount of analyte in a sample which could be detected; while, it is not necessarily quantitated as an exact value. Moreover, the limit of quantitation (QL) of an analytical approach is the lowest amount of analyte in a sample, which could be quantitatively determined with a suitable level of precision and accuracy. As given in following, in this study, LD and QL were determined based on the standard deviation of the $y$-intercept and the slope of the calibration curve. ${ }^{15}$

The LD and QL are expressed as (Eq. (1), and Eq. (2)):

$$
\begin{aligned}
& L O D=\frac{3.3 \sigma}{S}, \\
& L O Q=\frac{10 \sigma}{S},
\end{aligned}
$$

where, $\sigma$ is the standard deviation of $y$-intercept, and $\mathrm{S}$ is the slope of the calibration curve. The LD and QL values are presented in Table 3.

Table 2. LD and QL values of the method for mixed standards calculated by Eq. (1), and Eq. (2).

\begin{tabular}{ccccc}
\hline & 3NSA & MLZ & 4APh & Phenol \\
\hline Slope & 62788 & 118571 & 38095 & 55919 \\
Standard deviation of y-intercept & 5877.6 & 2166.6 & 3497.3 & 1690.6 \\
LD (mg/L) & 0.309 & 0.060 & 0.303 & 0.100 \\
QL (mg/L) & 0.936 & 0.183 & 0.918 & 0.302 \\
\hline
\end{tabular}

The results showed that the (LD; QL) values for 3NSA, MLZ, 4APh, and Phenol, are $(0.309 ; 0.936)$, $(0.060 ; 0.183),(0.303 ; 0.918)$, and $(0.100 ; 0.302)$, respectively; which indicate the method is properly sensitive. The acceptance limit for LD and QL is $0.5 \mathrm{mg} / \mathrm{L}, 1.0 \mathrm{mg} / \mathrm{L}$, respectively.

\subsubsection{Accuracy}

The accuracy of the method has been evaluated by means of analyzing mixed standard concentration levels. The recovery percentages were calculated regarding to the regression equation. The results show that the recovery data are in the acceptable range of 99.9-100.2\%. Accuracy is commonly reported as the percent recovery of the known, added amount, or as the difference between the mean and true value with confidence intervals. The acceptance limit for recovery data are from $99.5-100.5 \%$.

Table 3. The accuracy of the method by using the recovery data at $5 \mathrm{ppm}$

\begin{tabular}{ccccc}
\hline & 3NSA & MLZ & 4APh & Phenol \\
\hline Slope & 62788 & 118571 & 38095 & 55919 \\
Standard deviation of y-intercept & 5877.6 & 2166.6 & 3497.3 & 1690.6 \\
Area (based on Calibration curve) & 299201 & 589663 & 181401 & 274698 \\
Recovery\% & 99.9 & 100.0 & 100.2 & 99.9 \\
\hline
\end{tabular}

\subsubsection{Precision}

Due to the literature, the precision of an analytical method is based on the closeness of agreement between a series of measurements prepared from multiple sampling of the same homogeneous sample under a defined condition. Thus, the precision is the standard deviation, variance, or coefficient of variation of a series of measurements. ${ }^{16}$

\subsubsection{Repeatability}

Repeatability of the present method was assessed by analyzing the mixed standards at $100 \%$ of the test concentration. As given in Table 4 , the $\%$ RSD values for peak responses are less than $2.0 \%$ which could be acceptable. The acceptance limit for $\%$ RSD is $2.0 \%$. 
Table 4. The interday, and the intraday precision for the mixed standards

\begin{tabular}{ccccc}
\hline & 3NSA & MLZ & 4APh & Phenol \\
\hline First day (Run 1) & 619024 & 1180134 & 371968 & 560698 \\
First day (Run 2) & 624240 & 1187175 & 373721 & 563044 \\
First day (Run 3) & 621083 & 1195911 & 373165 & 562822 \\
Second day (Run 1) & 626286 & 1190157 & 380162 & 564115 \\
Second day (Run 2) & 627146 & 1180734 & 377869 & 566153 \\
Second day (Run 3) & 621165 & 1182584 & 373677 & 562913 \\
Average (First day) & 621449 & 1187740 & 372951 & 562188 \\
Average (Second day) & 624866 & 1184492 & 377236 & 564394 \\
RSD (First day) & 0.42 & 0.67 & 0.24 & 0.23 \\
RSD (Second day) & 0.52 & 0.56 & 0.43 & 0.25 \\
RSD (Intraday) & 0.58 & 0.58 & 0.58 & 0.58 \\
\hline
\end{tabular}

\section{Conclusions}

In this project, a reverse phase HPLC method for a very fast (about 6 minutes) and simple separation of mesalamine from 4-aminophenol, phenol, and 3-nitrosalicylic acid was developed. The chromatogram peaks refereed to the four mentioned compounds were mostly high resolution. The results showed that $0.01 \mu \mathrm{g} / 1(0.01 \mathrm{ppm})$ of mesalamine could be detected by a sharp-tiny peak, which shows the sensitivity of the method in detection of this compound. The method was shown to be robust within the defined design conditions. A cheap and usual C18 liquid chromatography column was used for the process, and the results showed that the mentioned column was suitable for the high-resolution separation of the considered compounds. The results showed that by increasing the methanol percentage, separation between peaks 3, and 4, increases; while peaks 1, and 2 become closer to each other and to the injection peak. Also, changing the $\mathrm{pH}$ of the buffer showed that it has significant effects on the peak separation, resolution, and retention times. It is probably due to amphoteric nature of all four considered compounds. Especially in the case of peaks 3, and 4, by decreasing the $\mathrm{pH}$ from about 5.5 to 2.5 , separation between those two has a great and observable increase. The linearity curve indicated that the coefficient of determination $\left(\mathrm{R}^{2}\right)$ for each of four standards were 0.999 which showed a good linearity in the concentration range of $0.1 \mathrm{ppm}$ to $10 \mathrm{ppm}$. Also, the results showed that the (LD;QL) values for 3NSA, MLZ, 4APh, and Phenol, are $(0.309 ; 0.936),(0.060 ; 0.183),(0.303 ; 0.918)$, and $(0.100 ; 0.302)$, respectively; which indicate the method is properly sensitive.

\section{Supplementary Data}

Supplementary data are available at the Journal home page.

\section{Acknowledgment}

Authors would like to thank Tofigh Daru Research and Engineering Company for financial and technical support of this work.

\section{Experimental}

Chemicals containing PDP, ortho-phosphoric acid (OPA), methanol, octane-1-sulfunic acid sodium salt (OSA), 4-Aminophenol (4APh), and phenol were prepared from Merck chemical company (Germany). Mesalamine (MLZ) and 3-nitrosalicylic acid (3NSA), compound was provided from the Chemical Synthesis Department of Tofigh Daru Research and Engineering Company (Tehran, Iran).

\subsection{Instrumentation}

The Shimadzu Prominence system (Shimadzu Corporation, Kyoto, Japan) equipped with a LC20AD pump, a DGU-20A degassing system, a CTO-20A column oven and a SPD-20A UV-Vis 
detector, was used for all analysis. Also, the LabSolutions software version 5.51 was applied for processing and data analysis.

\subsection{Chromatographic conditions and sample preparation}

In the optimized chromatography condition, a C18, end-capped $(250 \times 4.6) \mathrm{mm}, 5 \mu \mathrm{m}$ liquid chromatography column was used for the analysis. The mobile phase was a mixture of (buffer:methanol) with a ratio of (60:40). The buffer was prepared by dissolving $1.1 \mathrm{~g}$ of $(5 \mathrm{mM})$ OSA in 1 liter of water to $\mathrm{pH}=2.70$ with OPA. The isocratic elusion with a flow rate of $1.5 \mathrm{ml} / \mathrm{min}$ was set for the method. In addition, a UV detector on the wavelength of $210 \mathrm{~nm}$ was used for recording the chromatograms. The column oven temperature was maintained at $40^{\circ} \mathrm{C}$ and the volume of injection was $20 \mu \mathrm{L}$. Also, the mobile phase was used as the diluent. The sample solutions were prepared as following:

Test solution (a:1000ppm): Dissolve $10 \mathrm{mg}$ of each of the considered substances containing 3NSA, MLZ, 4APh, and Phenol the diluent (1000 $\mathrm{mg} / \mathrm{L})$.

Test solution (b:10ppm): Dilute $1.0 \mathrm{~mL}$ of Test solution (a) to $100.0 \mathrm{~mL}$ with the diluent (10 mg/L).

Test solution (c:5ppm): Dilute $5.0 \mathrm{~mL}$ of Test solution (b) to $10.0 \mathrm{~mL}$ with the diluent $(5 \mathrm{mg} / \mathrm{L})$.

Test solution (d:1ppm): Dilute $2.0 \mathrm{~mL}$ of Test solution (c) to $10.0 \mathrm{~mL}$ with the diluent $(1 \mathrm{mg} / \mathrm{L})$.

Test solution (e:0.5ppm): Dilute $5.0 \mathrm{~mL}$ of Test solution (d) to $10.0 \mathrm{~mL}$ with the diluent $(0.5 \mathrm{mg} / \mathrm{L})$.

Test solution (f:0.1ppm): Dilute $2.0 \mathrm{~mL}$ of Test solution (e) to $10.0 \mathrm{~mL}$ with the diluent $(0.1 \mathrm{mg} / \mathrm{L})$.

Test solution (g:0.01ppm): Dilute $1.0 \mathrm{~mL}$ of Test solution (f) to $10.0 \mathrm{~mL}$ with the diluent $(0.01 \mathrm{mg} / \mathrm{L})$.

The injections were $20 \mu \mathrm{L}$ of the Blank solution (which is the mobile phase, used as the diluent), the Test solutions (a) to $(\mathrm{g})$, respectively.

\section{References}

1 Bento-Silva A., Koistinen V. M., Mena P., Bronze M. R., Hanhineva K., Sahlstrom S., Aura A. M. (2019) Factors affecting intake, metabolism and health benefits of phenolic acids: do we understand individual variability?. Eur. J. Nutr. 59 (4) 1275-1293.

2 Wang L., Asimakopoulos A. G., Kannan K. (2015) Accumulation of 19 environmental phenolic and xenobiotic heterocyclic aromatic compounds in human adipose tissue, Environ. Int., 78, 45-50.

3 Zhang V., Xiaoqiu Y. U., Zhang Y., Cui X., Wang C. (2016) Determination of Aniline, 2Aminophenol and 4-Aminophenol in Mesalazine Sustained Release Granules by Gas Chromatography, Herald Med., 2, 185-188.

4 Song D. M., Yang Y. H. (2007) Determination of related substances in mesalazine by HPLCelectrochemical detection, Chin. J. Pharm., 38, 442.

5 Payab M., Beheshti A., Siadati S. A. (2020) Development of a reversed-phase HPLC method for determination of related impurities of Lenalidomide, Chem. Rev. Lett., 3 (2) 61-64.

6 UNITED States Pharmacopeia 24. ed. Rockville, United States Pharmacopeial Convention, (2000). [CD-ROOM].

7 United States Pharmacopoeia Convention, The United States Pharmacopoeia 36th ed. (USP 36). 2014, Rockville, Maryland, USA: United States Pharmacopeial Convention, Incorporated.

8 a) Grondal Ch., Jeanty M., Enders D. (2010) Organocatalytic cascade reactions as a new tool in total synthesis, Nature chem., 2 (3) 167-178; b) Jasiński, R., Dresler, E. (2020) On the Question of Zwitterionic Intermediates in the [3+2] Cycloaddition Reactions: A Critical Review. Organics, 1(1) 49-69; c) Siadati, S. A. (2015) An example of a stepwise mechanism for the catalyst-free 1, 3-dipolar cycloaddition between a nitrile oxide and an electron rich alkene. Tetrahedron let., 56 (34) 48574863; d) Siadati, S. A. (2016) Beyond the alternatives that switch the mechanism of the 1,3-dipolar cycloadditions from concerted to stepwise or vice versa: a literature review. Prog. React. Kinet. Mech., 41 (4) 331-344.

9 a) Bakherad M., Keivanloo A., Siavashi M., Omidian M. (2014) Three-component synthesis of imidazo [1, 2-c] pyrimidines using silica sulfuric acid (SSA), Chin. Chem. Lett., 25 (1) 149-151; b) 
Rostami-Charati F., Hossaini Z., Sheikholeslami-Farahani F., Azizi Z., Siadati, A. S. (2015) Synthesis of 9H-furo [2, 3-f] Chromene Derivatives by Promoting ZnO Nanoparticles. Comb. chem. high throughput screen., 18 (9) 872-880; c) Siadati, S. A. (2016) The Effect of Position Replacement of Functional Groups on the Stepwise character of 1, 3-Dipolar Reaction of a Nitrile Oxide and an Alkene. Helv. Chim. Acta, 99 (4) 273-280.

10 a) Bakherad M., Keivanloo A., Omidian M., Samangooei S. (2014) Synthesis of pyrrolo [2, 3-b] pyrazines through Sonogashira coupling reaction of 5, 6-dichloropyrazine-2, 3-dicarbonitrile with hydrazine, phenylacetylene and various aldehydes, J Chem. Res., 38 (12) 762-764; b) Hossaini Z., Rostami-Charati F., Ghambarian M., Siadati S. A. (2015) Synthesis of a new class of phosphonate derivatives using a three component reaction of trialkyl phosphites or triaryl phosphites in water. Phosphorus Sulfur Silicon Relat. Elem., 190 (7) 1177-1182.

11 D'Hondt M., Gevaert B., Stalmans S., Van Dorpe S., Wynendaele E., Peremans K., De Spiegeleer B. (2013) Reversed-phase fused-core HPLC modeling of peptides. J. pharm. anal., 3 (2) 93-101.

12 Schellinger A. P., Carr P. W. (2006) Isocratic and gradient elution chromatography: A comparison in terms of speed, retention reproducibility and quantization, J Chromatogr. A 1109 (2) 253-266.

13 Snyder L. R., Dolan J. W. (1996) Initial experiments in high-performance liquid chromatographic method development I. Use of a starting gradient run, J. Chromatogr. A, 721 (1) 3-14.

14 Hou J. J., Wu W. Y., Da J., Yao S., Long H. L., Yang Z., Guo D. A. (2011) Ruggedness and robustness of conversion factors in method of simultaneous determination of multi-components with single reference standard. J. Chromatogr., 1218 (33) 5618-5627.

15 Armbruster D. A., Tillman M. D., Hubbs L. M. (1994) Limit of detection (LQD)/limit of quantitation (LOQ): comparison of the empirical and the statistical methods exemplified with GC-MS assays of abused drugs. Clinical chem., 40 1233-1238.

16 Ermer J., Arth C., De Raeve P., Dill D., Friedel H. D., Höwer-Fritzen H., Maegerlein M. (2005) Precision from drug stability studies: Investigation of reliable repeatability and intermediate precision of HPLC assay procedures. J. Pharm. Biomed. Anal. 38 (4) 653-663.

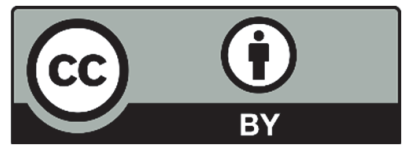

(C) 2021 by the authors; licensee Growing Science, Canada. This is an open access article distributed under the terms and conditions of the Creative Commons Attribution (CC-BY) license (http://creativecommons.org/licenses/by/4.0/). 